\title{
SOME NEW PROPERTIES ON THE $q$-GENOCCHI NUMBERS AND POLYNOMIALS ASSOCIATED WITH $q$-BERNSTEIN POLYNOMIALS
}

\author{
Serkan Araci, Dilek Erdal and Dong-Jin Kang
}

\begin{abstract}
The purpose of this study is to obtain some relations between $q$-Genocchi numbers and $q$-Bernstein polynomials by using fermionic $p$-adic $q$-integral on $\mathbb{Z}_{p}$.
\end{abstract}

\section{Introduction, Definitions and Notations}

Let $p$ be a fixed odd prime number. Throughout this paper we use the following notations. By $\mathbb{Z}_{p}$ we denote the ring of $p$-adic rational integers, $\mathbb{Q}$ denotes the field of rational numbers, $\mathbb{Q}_{p}$ denotes the field of $p$-adic rational numbers, and $\mathbb{C}_{p}$ denotes the completion of algebraic closure of $\mathbb{Q}_{p}$. Let $\mathbb{N}$ be the set of natural numbers and $\mathbb{N}^{*}=\mathbb{N} \cup\{0\}$. The $p$-adic absolute value is defined by $|x|_{p}=\frac{1}{p}$. In this paper we assume $|q-1|_{p}<1$ as an indeterminate. In [17]-[20], Kim defined the fermionic $p$-adic $q$-integral on $\mathbb{Z}_{p}$ as follows:

$$
I_{-q}(g)=\int_{\mathbb{Z}_{p}} g(x) d \mu_{-q}(x)=\lim _{N \rightarrow \infty} \frac{1}{\left[p^{N}\right]_{-q}} \sum_{x=0}^{p^{N}-1} g(x)(-q)^{x}
$$

For $k, n \in \mathbb{N}^{*}$ and $x \in[0,1]$, Kim's $q$-Bernstein polynomials are given as follows $([8])$ :

$$
B_{k, n}(x, q)=\left(\begin{array}{l}
n \\
k
\end{array}\right)[x]_{q}^{k}[1-x]_{q^{-1}}^{n-k}
$$

Received April 26, 2011. Accepted May 10, 2011.

2000 Mathematics Subject Classification. Primary 46A15, Secondary 41A65.

Key words and phrases. Genocchi numbers and polynomials, $q$-Genocchi numbers and polynomials, Bernstein polynomials, $q$-Bernstein polynomials. 
where, $[x]_{q}$ is a $q$-extension of $x$ defined by (see [1] $\left.-[22]\right)$ :

$$
[x]_{q}=\frac{1-q^{x}}{1-q}
$$

Note that $\lim _{q \rightarrow 1}[x]_{q}=x$.

For $n \in \mathbb{N}^{*}$, let us consider the $q$-Genocchi polynomials as follows:

$$
G_{n+1, q}(x)=(n+1) \int_{\mathbb{Z}_{p}}[x+y]_{q}^{n} d \mu_{-q}(y) .
$$

In the special case, $x=0, G_{n, q}(0)=G_{n, q}$ are called the $q$-Genocchi numbers.

In this paper we obtain some relations between the $q$-Bernstein polynomials and the $q$-Genocchi numbers. From these relations, we derive some interesting identities on the $q$-Genocchi numbers and polynomials.

\section{On the $q$-Genocchi numbers and $q$-Bernstein polynomials}

By the definition of $q$-Genocchi polynomials, we easily get

$$
\begin{aligned}
G_{n+1, q}(x) & =(n+1) \int_{\mathbb{Z}_{p}}[x+y]_{q}^{n} d \mu_{-q}(y) \\
& =(n+1) \frac{[2]_{q}}{(1-q)^{n}} \sum_{k=0}^{n}\left(\begin{array}{l}
n \\
k
\end{array}\right) q^{k x} \frac{(-1)^{k}}{1+q^{k+1}} \\
& =(n+1)[2]_{q} \sum_{l=0}^{\infty}(-1)^{l} q^{l}[x+l]_{q}^{n},
\end{aligned}
$$


and

$$
\begin{aligned}
\sum_{n=0}^{\infty} G_{n, q}(x) \frac{t^{n}}{n !} & =[2]_{q} t \sum_{n=0}^{\infty}(-1)^{n} q^{n} e^{[x+n]_{q} t} \\
& =[2]_{q} t \sum_{n=0}^{\infty}(-1)^{n} q^{n} e^{\left([x]_{q}+q^{x}[n]_{q}\right) t} \\
& =\left(\frac{e^{[x]_{q} t}}{q^{x}}\right)\left([2]_{q} q^{x} t \sum_{n=0}^{\infty}(-1)^{n} q^{n} e^{\left(q^{x} t\right)[n]_{q}}\right) \\
& =\left(\sum_{n=0}^{\infty}[x]_{q}^{n} \frac{t^{n}}{n !}\right)\left(\sum_{n=0}^{\infty} q^{(n-1) x} G_{n, q} \frac{t^{n}}{n !}\right) \\
& =\sum_{n=0}^{\infty}\left(\sum_{k=0}^{n}\left(\begin{array}{l}
n \\
k
\end{array}\right) q^{(k-1) x}[x]_{q}^{n-k} G_{k, q}\right) \frac{t^{n}}{n !}
\end{aligned}
$$

Therefore we obtain the following theorem.

Theorem 1. For $n \in \mathbb{N}^{*}$, we have

$$
\begin{aligned}
G_{n+1, q}(x) & =(n+1)[2]_{q} \sum_{l=0}^{\infty}(-1)^{l} q^{l}[x+l]_{q}^{n} \\
& =\sum_{k=0}^{n+1}\left(\begin{array}{c}
n+1 \\
k
\end{array}\right) q^{(k-1) x}[x]_{q}^{n+1-k} G_{k, q} \\
& =q^{-x}\left([x]_{q}+q^{x} G_{q}\right)^{n+1}
\end{aligned}
$$

with usual convention about replacing $\left(G_{q}\right)^{n}$ by $G_{n, q}$.

By Theorem 1, we have

$$
\sum_{n=0}^{\infty} G_{n, q}(x) \frac{t^{n}}{n !}=[2]_{q} t \sum_{n=0}^{\infty}(-1)^{n} q^{n} e^{[x+n]_{q} t} .
$$


By (2.1), we get

$$
\begin{aligned}
G_{n+1, q^{-1}}(1-x) & =(n+1) \int_{\mathbb{Z}_{p}}[1-x+y]_{q^{-1}}^{n} d \mu_{-q^{-1}}(y) \\
& =(n+1) \frac{1+q^{-1}}{\left(1-q^{-1}\right)^{n}} \sum_{k=0}^{n}\left(\begin{array}{l}
n \\
k
\end{array}\right) q^{-k(1-x)} \frac{(-1)^{k}}{1+q^{-k-1}} \\
& =(n+1)(-1)^{n} q^{n} \frac{[2]_{q}}{(1-q)^{n}} \sum_{k=0}^{n}\left(\begin{array}{l}
n \\
k
\end{array}\right) q^{k x} \frac{(-1)^{k}}{1+q^{k+1}} \\
& =(-1)^{n} q^{n} G_{n+1, q}(x) .
\end{aligned}
$$

Therefore, we obtain the following theorem.

Theorem 2. For $n \in \mathbb{N}^{*}$, we have

$$
G_{n, q^{-1}}(1-x)=(-1)^{n-1} q^{n-1} G_{n, q}(x) .
$$

From (2.1) we note that

$$
q \sum_{n=0}^{\infty} G_{n, q}(1) \frac{t^{n}}{n !}+\sum_{n=0}^{\infty} G_{n, q} \frac{t^{n}}{n !}=[2]_{q} t .
$$

By (2.2), we get the following recurrence formula:

$$
G_{0, q}=0, q G_{n, q}(1)+G_{n, q}= \begin{cases}{[2]_{q},} & n=1 \\ 0, & n>1\end{cases}
$$

From (2.3) and Theorem 1, we have the following theorem.

Theorem 3. For $n \in \mathbb{N}^{*}$, we have

$$
G_{0, q}=0, \text { and } q\left(q G_{q}+1\right)^{n}+G_{n, q}= \begin{cases}{[2]_{q},} & n=1 \\ 0, & n>1\end{cases}
$$

with usual convention about replacing $\left(G_{q}\right)^{n}$ by $G_{n, q}$.

For $n \in \mathbb{N}$, by Theorem 3, we note that

$$
\begin{aligned}
q^{-2} G_{n, q}(2) & =q^{-2} \sum_{l=0}^{n}\left(\begin{array}{c}
n \\
l
\end{array}\right) q^{l}\left(q G_{q}+1\right)^{l} \\
& =n q^{-2}\left([2]_{q}-G_{1, q}\right)+q^{-2} \sum_{l=0}^{n}\left(\begin{array}{c}
n \\
l
\end{array}\right) q^{l} G_{l, q}(1) \\
& =n q^{-1}-q^{-3} \sum_{l=0}^{n}\left(\begin{array}{c}
n \\
l
\end{array}\right) q^{l} G_{l, q} \\
& =n q^{-1}+q^{-4} G_{n, q}, \text { if } n>1
\end{aligned}
$$


Therefore, we have the following theorem.

Theorem 4. For $n \in \mathbb{N}$, we have

$$
G_{n+1, q}(2)=q^{-2} G_{n+1, q}+(n+1) q .
$$

From Theorem 2, we see that

$$
\begin{aligned}
& (n+1) \int_{\mathbb{Z}_{p}}[1-x]_{q^{-1}}^{n} d \mu_{-q}(x) \\
= & (-q)^{n}(n+1) \int_{\mathbb{Z}_{p}}[x-1]_{q}^{n} d \mu_{-q}(x) \\
= & (-1)^{n} q^{n} G_{n+1, q}(-1)=G_{n+1, q^{-1}}(2) .
\end{aligned}
$$

Therefore, we have the following theorem.

Theorem 5. For $n \in \mathbb{N}^{*}$, we have

$$
(n+1) \int_{\mathbb{Z}_{p}}[1-x]_{q^{-1}}^{n} d \mu_{-q}(x)=G_{n+1, q^{-1}}(2) .
$$

Let $n \in \mathbb{N}$. By Theorem 4 and Theorem 5 , we get

$$
\begin{aligned}
& (n+1) \int_{\mathbb{Z}_{p}}[1-x]_{q^{-1}}^{n} d \mu_{-q}(x) \\
= & q^{2} G_{n+1, q^{-1}}+\frac{(n+1)}{q}
\end{aligned}
$$

For (2.5), we obtain

$$
(n+1) \int_{\mathbb{Z}_{p}}[1-x]_{q^{-1}}^{n} d \mu_{-q}(x)=q^{2} G_{n+1, q^{-1}}+\frac{(n+1)}{q} .
$$

Corollary 1. For $n \in \mathbb{N}^{*}$, we have

$$
\int_{\mathbb{Z}_{p}}[1-x]_{q^{-1}}^{n} d \mu_{-q}(x)=\frac{q^{2}}{n+1} G_{n+1, q^{-1}}+\frac{1}{q} .
$$

\section{New identities on the $q$-Genocchi numbers and $q$-Genocchi polynomials}

In this section, we give some interesting relationship between the $q$-Genocchi numbers and $q$-Bernstein polynomials. given by

For $x \in \mathbb{Z}_{p}$, the $p$-adic analogues of $q$-Bernstein polynomials are

$$
B_{k, n}(x, q)=\left(\begin{array}{l}
n \\
k
\end{array}\right)[x]_{q}^{k}[1-x]_{q^{-1}}^{n-k}, \text { where } n, k \in \mathbb{N}^{*} .
$$


By (3.1), Kim [10] gave the symmetry of $q$-Bernstein polynomials as follows:

$$
B_{k, n}(x, q)=B_{n-k, n}\left(1-x, q^{-1}\right),
$$

Thus, from Corollary 6, (3.1) and (3.2), we see that

$$
\begin{aligned}
\int_{\mathbb{Z}_{p}} B_{k, n}(x, q) d \mu_{-q}(x) & =\int_{\mathbb{Z}_{p}} B_{n-k, n}\left(1-x, q^{-1}\right) d \mu_{-q}(x) \\
& =\left(\begin{array}{l}
n \\
k
\end{array}\right) \sum_{l=0}^{k}\left(\begin{array}{l}
k \\
l
\end{array}\right)(-1)^{k+l} \int_{\mathbb{Z}_{p}}[1-x]_{q^{-1}}^{n-l} d \mu_{-q}(x) \\
& =\left(\begin{array}{l}
n \\
k
\end{array}\right) \sum_{l=0}^{k}\left(\begin{array}{l}
k \\
l
\end{array}\right)(-1)^{k+l}\left(\frac{q^{2}}{n-l+1} G_{n-l+1, q^{-1}}+\frac{1}{q}\right) .
\end{aligned}
$$

For $n, k \in \mathbb{N}^{*}$ with $n>k$, we obtain

$$
\begin{aligned}
& \int_{\mathbb{Z}_{p}} B_{k, n}(x, q) d \mu_{-q}(x) \\
(3.3)= & \left(\begin{array}{l}
n \\
k
\end{array}\right) \sum_{l=0}^{k}\left(\begin{array}{l}
k \\
l
\end{array}\right)(-1)^{k+l}\left(\frac{q^{2}}{n-l+1} G_{n-l+1, q^{-1}}+\frac{1}{q}\right) \\
= & \begin{cases}\frac{q^{2}}{n+1} G_{n+1, q^{-1}}+\frac{1}{q}, \\
\left(\begin{array}{l}
n \\
k
\end{array}\right) \sum_{l=0}^{k}\left(\begin{array}{l}
k \\
l
\end{array}\right)(-1)^{k+l}\left(\frac{q^{2}}{n-l+1} G_{n-l+1, q^{-1}}+\frac{1}{q}\right), & \text { if } k>0,\end{cases}
\end{aligned}
$$

Let us take the fermionic $q$-integral on $\mathbb{Z}_{p}$ for the $q$-Bernstein polynomials of degree $n$ as follows:

$$
\begin{aligned}
(3.4) \int_{\mathbb{Z}_{p}} B_{k, n}(x, q) d \mu_{-q}(x) & =\left(\begin{array}{l}
n \\
k
\end{array}\right) \int_{\mathbb{Z}_{p}}[x]_{q}^{k}[1-x]_{q^{-1}}^{n-k} d \mu_{-q}(x) \\
& =\left(\begin{array}{l}
n \\
k
\end{array}\right) \sum_{l=0}^{n-k}\left(\begin{array}{c}
n-k \\
l
\end{array}\right)(-1)^{l} \frac{G_{l+k+1, q}}{l+k+1} .
\end{aligned}
$$

Therefore, by (3.3) and (3.4), we obtain the following theorem.

Theorem 6. For $n, k \in \mathbb{N}^{*}$ with $n>k$. we have

$$
\sum_{l=0}^{n-k}\left(\begin{array}{c}
n-k \\
l
\end{array}\right)(-1)^{l} \frac{G_{l+k+1, q}}{l+k+1}= \begin{cases}\frac{q^{2}}{n+1} G_{n+1, q^{-1}}+\frac{1}{q}, & \text { if } k=0, \\
\sum_{l=0}^{k}\left(\begin{array}{l}
k \\
l
\end{array}\right)(-1)^{k+l}\left(\frac{q^{2}}{n-l+1} G_{n-l+1, q^{-1}}+\frac{1}{q}\right), & \text { if } k>0 .\end{cases}
$$


Let $n_{1}, n_{2}, k \in \mathbb{N}^{*}$ with $n_{1}+n_{2}>2 k$. Then we get

$$
\begin{aligned}
& \int_{\mathbb{Z}_{p}} B_{k, n_{1}}(x, q) B_{k, n_{2}}(x, q) d \mu_{-q}(x) \\
= & \left(\begin{array}{c}
n_{1} \\
k
\end{array}\right)\left(\begin{array}{c}
n_{2} \\
k
\end{array}\right) \sum_{l=0}^{2 k}\left(\begin{array}{c}
2 k \\
l
\end{array}\right)(-1)^{2 k+l} \int_{\mathbb{Z}_{p}}[1-x]_{q^{-1}}^{n_{1}+n_{2}-l} d \mu_{-q}(x) \\
= & \left(\begin{array}{c}
n_{1} \\
k
\end{array}\right)\left(\begin{array}{c}
n_{2} \\
k
\end{array}\right) \sum_{l=0}^{2 k}\left(\begin{array}{c}
2 k \\
l
\end{array}\right)(-1)^{2 k+l}\left(\frac{q^{2}}{n_{1}+n_{2}-l+1} G_{n_{1}+n_{2}-l+1, q^{-1}}+\frac{1}{q}\right) \\
= & \left\{\begin{array}{cc}
\frac{q^{2}}{n_{1}+n_{2}+1} G_{n_{1}+n_{2}+1, q^{-1}+\frac{1}{q},} & \text { if } k=0, \\
\left(\begin{array}{c}
n_{1} \\
k
\end{array}\right)\left(\begin{array}{c}
n_{2} \\
k
\end{array}\right) \sum_{l=0}^{2 k}\left(\begin{array}{c}
2 k \\
l
\end{array}\right)(-1)^{2 k+l}\left(\frac{q^{2}}{n_{1}+n_{2}-l+1} G_{n_{1}+n_{2}-l+1, q^{-1}}+\frac{1}{q}\right), & \text { if } k \neq 0 .
\end{array}\right.
\end{aligned}
$$

Therefore, we obtain the following theorem.

Theorem 7. For $n_{1}, n_{2}, k \in \mathbb{N}^{*}$ with $n_{1}+n_{2}>2 k$, we have

$$
\begin{aligned}
& \int_{\mathbb{Z}_{p}} B_{k, n_{1}}(x, q) B_{k, n_{2}}(x, q) d \mu_{-q}(x) \\
= & \begin{cases}\frac{q^{2}}{n_{1}+n_{2}+1} G_{n_{1}+n_{2}+1, q^{-1}}+\frac{1}{q}, & \text { if } k=0, \\
\left(\begin{array}{c}
n_{1} \\
k
\end{array}\right)\left(\begin{array}{c}
n_{2} \\
k
\end{array}\right) \sum_{l=0}^{2 k}\left(\begin{array}{c}
2 k \\
l
\end{array}\right)(-1)^{2 k+l}\left(\frac{q^{2}}{n_{1}+n_{2}-l+1} G_{n_{1}+n_{2}-l+1, q^{-1}}+\frac{1}{q}\right), & \text { if } k \neq 0 .\end{cases}
\end{aligned}
$$

From the binomial theorem, we can derive the following equation.

$$
\begin{aligned}
& \int_{\mathbb{Z}_{p}} B_{k, n_{1}}(x, q) B_{k, n_{2}}(x, q) d \mu_{-q}(x) \\
(3.5)= & \prod_{i=1}^{2}\left(\begin{array}{c}
n_{i} \\
k
\end{array}\right) \sum_{l=0}^{n_{1}+n_{2}-2 k}\left(\begin{array}{c}
n_{1}+n_{2}-2 k \\
l
\end{array}\right)(-1)^{l} \int_{\mathbb{Z}_{p}}[x]_{q}^{2 k+l} d \mu_{-q}(x) \\
= & \prod_{i=1}^{2}\left(\begin{array}{c}
n_{i} \\
k
\end{array}\right) \sum_{l=0}^{n_{1}+n_{2}-2 k}\left(\begin{array}{c}
n_{1}+n_{2}-2 k \\
l
\end{array}\right)(-1)^{l} \frac{G_{l+2 k+1, q}}{l+2 k+1} .
\end{aligned}
$$

Thus, for theorem 8 and (3.6), we obtain the following corollary.

Corollary 2. Let $n_{1}, n_{2}, k \in \mathbb{N}^{*}$ with $n_{1}+n_{2}>2 k$, we have

$$
\begin{aligned}
& \sum_{l=0}^{n_{1}+n_{2}-2 k}\left(\begin{array}{c}
n_{1}+n_{2}-2 k \\
l
\end{array}\right)(-1)^{l} \frac{G_{l+2 k+1, q}}{l+2 k+1} \\
= & \begin{cases}\frac{q^{2}}{n_{1}+n_{2}+1} G_{n_{1}+n_{2}+1, q^{-1}}+\frac{1}{q}, & \text { if } k=0 \\
\sum_{l=0}^{2 k}\left(\begin{array}{c}
2 k \\
l
\end{array}\right)(-1)^{2 k+l}\left(\frac{q^{2}}{n_{1}+n_{2}-l+1} G_{n_{1}+n_{2}-l+1, q^{-1}}+\frac{1}{q}\right), & \text { if } k \neq 0 .\end{cases}
\end{aligned}
$$


For $x \in \mathbb{Z}_{p}$ and $s \in \mathbb{N}$ with $s \geq 2$, let $n_{1}, n_{2}, \ldots, n_{s}, k \in \mathbb{N}^{*}$ with $\sum_{l=1}^{s} n_{l}>s k$. Then we take the fermionic $p$-adic $q$-integral on $\mathbb{Z}_{p}$ for the $q$-Bernstein polynomials of degree $n$ as follows:

$$
\begin{aligned}
& \int_{\mathbb{Z}_{p}} \underbrace{B_{k, n_{1}}(x, q) B_{k, n_{2}}(x, q) \ldots B_{k, n_{s}}(x, q)}_{s-t i m e s} d \mu_{-q}(x) \\
= & \prod_{i=1}^{s}\left(\begin{array}{c}
n_{i} \\
k
\end{array}\right) \int_{\mathbb{Z}_{p}}[x]_{q}^{s k}[1-x]_{q^{-1}}^{n_{1}+n_{2}+\ldots+n_{s}-s k} d \mu_{-q}(x) \\
= & \prod_{i=1}^{s}\left(\begin{array}{c}
n_{i} \\
k
\end{array}\right) \sum_{l=0}^{s k}\left(\begin{array}{c}
s k \\
l
\end{array}\right)(-1)^{l+s k} \int_{\mathbb{Z}_{p}}[1-x]_{q^{-1}}^{n_{1}+n_{2}+\ldots+n_{s}-s k} d \mu_{-q}(x) \\
= & \begin{cases}\frac{q^{2}}{n_{1}+\ldots n_{s}+1} G_{n_{1}+\ldots+n_{s}+1, q-1}+\frac{1}{q}, & \text { if } k=0, \\
\prod_{i=1}^{s}\left(\begin{array}{c}
n_{i} \\
k
\end{array}\right) \sum_{l=0}^{2 k}\left(\begin{array}{c}
2 k \\
l
\end{array}\right)(-1)^{2 k+l}\left(\frac{q^{2}}{n_{1}+\ldots+n_{s}-l+1} G_{n_{1}+\ldots+n_{s}-l+1, q^{-1}}+\frac{1}{q}\right), & \text { if } k \neq 0 .\end{cases}
\end{aligned}
$$

Therefore, we obtain the following theorem.

Theorem 8. For $s \in \mathbb{N}$ with $s \geq 2$, let $n_{1}, n_{2}, \ldots, n_{s}, k \in \mathbb{N}^{*}$ with $\sum_{l=1}^{s} n_{l}>s k$. Then we have

$$
\begin{aligned}
& \int_{\mathbb{Z}_{p}} \prod_{i=1}^{s} B_{k, n_{i}}(x) d \mu_{-q}(x) \\
& = \begin{cases}\frac{q^{2}}{n_{1}+\ldots n_{s}+1} G_{n_{1}+\ldots n_{s}+1, q^{-1}}+\frac{1}{q}, & \text { if } k=0, \\
\prod_{i=1}^{s}\left(\begin{array}{c}
n_{i} \\
s
\end{array}\right) \sum_{l=0}^{s k}\left(\begin{array}{c}
s k \\
l
\end{array}\right)(-1)^{s k+l}\left(\frac{q^{2}}{n_{1}+\ldots+n_{s}-l+1} G_{n_{1}+\ldots+n_{s}-l+1, q^{-1}}+\frac{1}{q}\right), & \text { if } k \neq 0 .\end{cases}
\end{aligned}
$$

From the definition of $q$-Bernstein polynomials and the binomial theorem, we easily get

$$
\begin{aligned}
& \int_{\mathbb{Z}_{p}} \underbrace{B_{k, n_{1}}(x, q) B_{k, n_{2}}(x, q) \ldots B_{k, n_{s}}(x, q)}_{s-\text { times }} d \mu_{-q}(x) \\
= & \prod_{i=1}^{s}\left(\begin{array}{c}
n_{i} \\
k
\end{array}\right) \sum_{l=0}^{n_{1}+\ldots+n_{s}-s k}\left(\begin{array}{c}
\sum_{d=1}^{s}\left(n_{d}-k\right) \\
l
\end{array}\right)(-1)^{l} \int_{\mathbb{Z}_{p}}[x]_{q}^{s k+l} d \mu_{-q}(x) \\
(3.6)= & \prod_{i=1}^{s}\left(\begin{array}{c}
n_{i} \\
k
\end{array}\right) \sum_{l=1}^{n_{1}+\ldots+n_{s}-s k}\left(\begin{array}{c}
\sum_{d=1}^{s}\left(n_{d}-k\right) \\
l
\end{array}\right)(-1)^{l} \frac{G_{l+s k+1, q}}{l+s k+1} .
\end{aligned}
$$

Therefore, from (3.6) and Theorem 10. we have the following corollary.

Corollary 3. For $s \in \mathbb{N}$ with $s \geq 2$, let $n_{1}, n_{2}, \ldots, n_{s}, k \in \mathbb{N}^{*}$ with $\sum_{l=1}^{s} n_{l}>s k$. we have

$$
\begin{aligned}
& \sum_{l=1}^{n_{1}+\ldots+n_{s}-s k}\left(\begin{array}{c}
\sum_{d=1}^{s}\left(n_{d}-k\right) \\
l
\end{array}\right)(-1)^{l} \frac{G_{l+s k+1, q}}{l+s k+1} \\
& = \begin{cases}\frac{q^{2}}{n_{1}+\ldots+n_{s}+1} G_{n_{1}+\ldots+n_{s}+1, q^{-1}}+\frac{1}{q}, & \text { if } k=0, \\
\sum_{l=0}^{s k}\left(\begin{array}{c}
s k \\
l
\end{array}\right)(-1)^{s k+l}\left(\frac{q^{2}}{n_{1}+\ldots n_{s}-l+1} G_{n_{1}+\ldots+n_{s}-l+1, q^{-1}}+\frac{1}{q}\right), & \text { if } k \neq 0 .\end{cases}
\end{aligned}
$$




\section{References}

[1] Açıkgöz, M. and Şimşek, Y., A New generating function of $q$-Bernstein type polynomials and their interpolation function, Abstract and Applied Analysis, Article ID 769095, 12 pages, doi: 10.1155/2010/769095.01-313.

[2] Açılkgöz, M., Aracı, S. and Cangül, N. İ., A Note on the modified $q$ Bernstein polynomials for functions of several variables and their reflections on $q$-Volkenborn integration, Applied Mathematics and Computation(in press)

[3] Gouvea, F. Q., p-adic Numbers An Introduction, 2nd Edi., Springer-Verlag, Berlin, Heidelberg and New York, 2000.

[4] Kim, T., A New Approach to q-Zeta Function, Adv. Stud. Contemp. Math. 11 (2) $157-162$

[5] Kim, T., On the $q$-extension of Euler and Genocchi numbers, J. Math. Anal. Appl. 326 (2007) 1458-1465.

[6] Kim, T., On the multiple $q$-Genocchi and Euler numbers, Russian J. Math. Phys. 15 (4) (2008) 481-486. arXiv:0801.0978v1 [math.NT]

[7] Kim, T., A Note on the $q$-Genocchi Numbers and Polynomials, Journal of Inequalities and Applications 2007 (2007) doi:10.1155/2007/71452. Article ID 71452,8 pages.

[8] Kim, T., A note $q$-Bernstein polynomials, Russ. J. Math. phys. 18(2011), 41-50.

[9] Kim, T., q- Volkenborn integration, Russ. J. Math. phys. 9(2002), 288-299.

[10] Kim, T., q-Bernoulli numbers and polynomials associated with Gaussian binomial coefficients, Russ. J. Math. Phys. 15(2008), 51-57.

[11] Kim, T. Choi, J. Kim, Y. H. Ryoo, C. S., On the fermionic p-adic integral representation of Bernstein polynomials associated with Euler numbers and polynomials, J. Inequal. Appl. 2010(2010), Art ID 864247, 12pp.

[12] Kim, T. Choi, J. Kim, Y. H., Some identities on the $q$-Bernstein polynomials, $q$-Stirling numbers and $q$-Bernoulli numbers, Adv. Stud. Contemp. Math. 20(2010), 335-341.

[13] Kim, T., An invariant $p$-adic $q$-integrals on $\mathbb{Z}_{p}$, Applied Mathematics Letters, vol. 21, pp. 105-108,2008.

[14] Kim, T. Choi, J. Kim, Y. H., q-Bernstein Polynomials Associated with $q$-Stirling Numbers and Carlitz's $q$-Bernoulli Numbers, Abstract and Applied Analysis, Article ID 150975, 11 pages, doi:10.1155/2010/150975

[15] Kim, T., A Note on the $q$-Genocchi Numbers and Polynomials, Journal of Inequalities and Applications, Article ID 71452, 8 pages, doi:10.1155/2007/71452.

[16] Kim, T. Choi, J. Kim, Y. H. and Jang, L. C., On $p$-Adic Analogue of $q$-Bernstein Polynomials and Related Integrals, Discrete Dynamics in Nature and Society, Article ID 179430, 9 pages, doi:10.1155/2010/179430.

[17] Kim, T., $q$-Euler numbers and polynomials associated with $p$-adic $q$-integrals, J. Nonlinear Math. Phys., 14 (2007), no. 1, 15-27.

[18] Kim, T., New approach to $q$-Euler polynomials of higher order, Russ. J. Math. Phys., 17 (2010), no. 2, 218-225.

[19] Kim, T., Some identities on the $q$-Euler polynomials of higher order and $q$-Stirling numbers by the fermionic $p$-adic integral on $\mathbb{Z}_{p}$, Russ. J. Math. Phys., 16 (2009), no.4,484-491.

[20] Kim, T., $q$-Bernoulli numbers and polynomials associated with Gaussian binomial coefficients, Russ. J. Math. Phys., 15 (2008), no. 1, 51-57. 
[21] Rim, S-H. Jin, J-H. Moon, E-J. and Lee, Sun-Jung., On Multiple Interpolation Functions of the $q$-Genocchi Polynomials, Journal of Inequalities and Applications, Article ID 13 pages, doi:10.1155/2010/351419

[22] Simsek, Y., Twisted $(h, q)$-Bernoulli numbers and polynomials related to twisted $(h, q)$-zeta function and L-function, Journal of Mathematical Analysis and Application, vol. 324, no. 2, pp. 790-804, 2006.

[23] Jang, L-C. Ryoo, C-S., A note on the Multiple Twisted Carlitz's Type qBernoulli Polynomials, Abstract and Applied Analysis, Article ID 498173, 7 pages, doi:10.1155/2008/498173.

Serkan Aracı

Department of Mathematics, University of Gaziantep, Faculty of Science and Arts,

Gaziantep 27310, Turkey.

E-mail: mtsrkn@hotmail.com

Dilek Erdal

Department of Mathematics, University of Gaziantep, Faculty of Science and Arts,

Gaziantep 27310, Turkey.

E-mail: dilekvecii@mynet.com

Dong-Jin Kang

Information Technology Service, Kyungpook National University,

Taegu 702-701, Korea.

E-mail: djkang@knu.ac.kr 